\title{
Visual and tactile memory for 2-D patterns: Effects of changes in size and left-right orientation
}

\author{
KAVITHA SRINIVAS, ANTHONY J. GREENE, and RANDOLPH D. EASTON \\ Boston College, Chestnut Hill, Massachusetts
}

\begin{abstract}
Visual identification of 3-D objects depends on representations that are invariant across changes in size and left-right orientation. We examined whether this finding reflects the unique demands of processing 3-D objects, or whether it generalizes to 2-D patterns and to the tactile modality. Our findings suggest that object representation for identification is influenced greatly by the processing demands of stimulus materials (e.g., 2-D vs. 3-D objects) and stimulus modality (touch vs. vision). Identification of 2-D patterns in vision is adversely affected by left-right orientation changes, but not size changes. Identification of the same patterns in touch is adversely affected by both changes. Together, the results suggest that the unique processing demands of stimulus materials and modality shape the representation of objects in memory.
\end{abstract}

How do we represent objects in memory? The answer to this question appears to differ, depending on whether memory is measured through explicit measures that require episodic recognition or through perceptual implicit measures that rely on the identification of an object. On explicit measures, memory is measured by asking participants to recollect a prior encounter with the object (e.g., asking participants whether an object has appeared earlier in the study phase of the experiment). On perceptual implicit measures, memory is measured by facilitation in the identification of an object due to a prior encounter with the object (e.g., asking participants to name an object after exposure to it in the study phase of the experiment). The two measures appear to tap different memory representations of objects.

Episodic recognition, for instance, appears to include information about object meaning such that elaborative processing at study (generating functions of objects) greatly enhances memory in comparison with the processing of physical features (counting vertices, or the number of vertical lines in an object; see Schacter, L. A. Cooper, \& Delaney, 1990). Identification measures, on the other hand, are unaffected by access to object meaning such that elaborative processing of an object or impairments in access to object meaning have no effect on facilitation (Srinivas, Breedin, Coslett, \& Saffran, 1997).

In addition to this difference in access to meaning, recognition and identification differ in their sensitivity to perceptual information about objects. Recognition of visual objects is adversely affected by "low-level" perceptual changes in an object's size, left-right orientation, position, contrast, illumination, color, and texture (Biederman \& E. E. Cooper, 1991, 1992; Cave, Bost, \& Cobb, 1996; Cave \& Squire, 1992; L. A. Cooper, Schacter,

Correspondence should be addressed to $K$. Srinivas, Department of Psychology, Boston College, Chestnut Hill, MA 02167-3807 (e-mail: kavitha.srinivas@bc.edu).
Ballesteros, \& Moore, 1992; Jolicoeur, 1987; Milliken \& Jolicoeur, 1992; Srinivas, 1996a, 1996b). Identification, on the other hand, is unaffected by these changes.

Why do identification and episodic recognition tap different types of information about objects? A common interpretation of their differences is that identification taps a perceptual system that computes presemantic, "structural descriptions" of objects that abstract across irrelevant changes in the size, left-right orientation, illumination, or position of the object (Biederman \& E. E. Cooper, 1992; L. A. Cooper et al., 1992). In contrast, episodic recognition is assumed to tap a declarative or episodic system that integrates shape information with an object's distinctive semantic or perceptual attributes (L. A. Cooper et al., 1992), presumably because these attributes can provide important cues for placing an object in a prior spatiotemporal context (Srinivas, 1996a, 1996b).

A somewhat different perspective on this issue is provided by an extension of transfer-appropriate processing approach, which states that the computational goals of the observer at study or at test govern the nature of object representations (Roediger \& Srinivas, 1993; Srinivas, 1996b). Within this view, identification is typically unaffected by perceptual changes (such as alteration of size or left-right orientation) because they usually reflect accidental changes (i.e., they occur because of an arbitrary alteration of observer position). However, there are instances in which the same perceptual changes may be relevant for identification. For instance, size changes may be important for certain object discriminations (e.g., discriminating a golf ball from a ping-pong ball), as are changes in left-right orientation (e.g., a $b$ differs from a $d$ in terms of its left-right orientation). According to the transfer-appropriate processing view, when size or leftright orientation is relevant for identification, it is likely to be included in representations in memory.

Initial support for the transfer-appropriate processing approach is provided in Srinivas (1996b), who found that 
size changes of familiar objects did affect visual identification when size was explicitly processed by participants at encoding. Left-right orientation changes of the same familiar objects, however, had no effect on visual identification, even when participants explicitly encoded this information at study. Srinivas (1996b) argued that these findings simply reflected the differential importance of size and left-right orientation information to the identification of 3-D familiar objects. Size information can sometimes be useful for discrimination of 3-D objects (e.g., in discriminating a golf ball from a ping-pong ball), and hence can be optionally represented for identification. Left-right orientation, on the other hand, is only useful for 2-D letter identification because it alters object identity for 2-D patterns but not for 3-D objects (e.g., in the discrimination of a $d$ from a $b$, or a $q$ from a $p$ ). Leftright orientation may therefore be expected to affect 2-D pattern identification rather than 3-D object identification (Roediger \& Srinivas, 1993; Srinivas, 1996b).

The present study is designed to extend the transferappropriate processing approach to the identification of visual and tactile 2-D objects. Consider, first, the predictions of the approach regarding visual identification. According to the transfer-appropriate processing approach, size and left-right orientation changes will affect identification measures if they cue a change in object identity. As indicated earlier, size changes can cue an alteration in object identity for some 3-D objects, but left-right orientation is almost always an accidental property of 3-D objects (i.e., it usually reflects a change analogous to a change in observer position in depth). For 2-D objects, however, the situation is reversed. Size changes do not cue a change in pattern identity, but left-right orientation is a critical nonaccidental property for discriminating patterns (a $p$ vs. a $q$ ). Thus, the transfer-appropriate processing approach would predict that size changes should not affect 2-D identification, but that left-right orientation changes should.

Now consider the predictions of the transfer-appropriate processing approach for identification of 2-D tactile objects. For reasons outlined above, tactile 2-D identification as well should be affected adversely by left-right orientation changes, because left-right orientation is an important cue to object identity for 2-D patterns. Tactile identification, in contrast to visual identification, should be adversely affected by size changes as well. In visual identification, size is often an accidental property, because object size is influenced by the distance of the observer with respect to the object. For tactile identification, on the other hand, this relationship between size and distance is no longer relevant. Size is therefore not an accidental property for tactile identification in the same sense that it is for vision, so it should affect tactile identification adversely.

We examined these predictions by contrasting the effects of size and left-right orientation alterations on visual and tactile 2-D identification. If size and left-right orientation alterations have no effect on visual and tactile 2-D identification, the results would suggest that 2-D patterns, like 3-D objects, are coded as abstract, structural descriptions of objects. If, on the other hand, leftright orientation alteration has an effect on 2-D identification for visual and tactile patterns, the results would suggest that the computational goals of identification greatly influence object representation. Finally, if size alteration affects tactile, but not visual, identification, the results would suggest that the goals of identification vary across modalitites. A comparison of vision and touch in the present experiments should also allow us to address the issue of whether the two perceptual systems represent perceptual information in an equivalent manner in memory, and this question, to our knowledge, has never been addressed in the literature.

We also included visual and tactile episodic recognition measures in our study, in order to address the issue of whether episodic recognition taps integrated representations of an object's shape along with distinctive perceptual attributes of objects (such as size or left-right orientation) regardless of the modality of the object. This allowed us to test the generality of the assertion that episodic recognition includes low-level perceptual information about objects that enables one to discriminate a prior encounter with the object from several other encounters with the same or similar objects (Srinivas, 1996b). In Experiment 1 , we therefore addressed the effects of size and left-right orientation changes on visual identification and episodic recognition. In Experiment 2, we examined the effects of size and left-right orientation changes on tactile identification and episodic recognition.

\section{EXPERIMENT 1}

\section{Method}

Participants. Ninety-six Boston College undergraduates participated in this experiment in partial fulfillment of a course requirement.

Materials and Design. A 2 (perceptual change: size or orientation, manipulated between subjects) $\times 3$ (study status: same, different, or nonstudied, manipulated within subjects) $\times 2$ (memory measure: visual identification or episodic recognition, manipulated between subjects) was used. Thirty students each were assigned to visual identification in the size change and orientation change conditions. An additional group of 18 each took part in the size change and orientation conditions of the visual recognition test. The stimuli were simple three-line patterns (approximately $.75 \times 2.25$ in. for small patterns and $1.5 \times 4.5$ in. for large patterns) made up of horizontal, vertical, or oblique line segments, following a procedure by Musen and Treisman (1990). These were presented on the computer. Thirty shapes were (see Figure 1 for sample shapes) and divided into blocks of 10 each. Each block of items was rotated through the three study conditions for counterbalancing. In the size change condition, of the 10 items presented in the same condition, half were presented in a large size at study and at test, and half were presented in a small size at study and at test. For the different items as well, half were presented large at study and small at test, and the other half were presented small at study and large at test. In the orientation change condition, of the 20 items presented in the same and different conditions, half were presented facing left at test, and the other half faced right.

Procedure. At study, participants were presented with 20 threeline shapes and were asked to describe the orientation of the lines (vertical, horizontal, or diagonal) and describe the type of junc- 


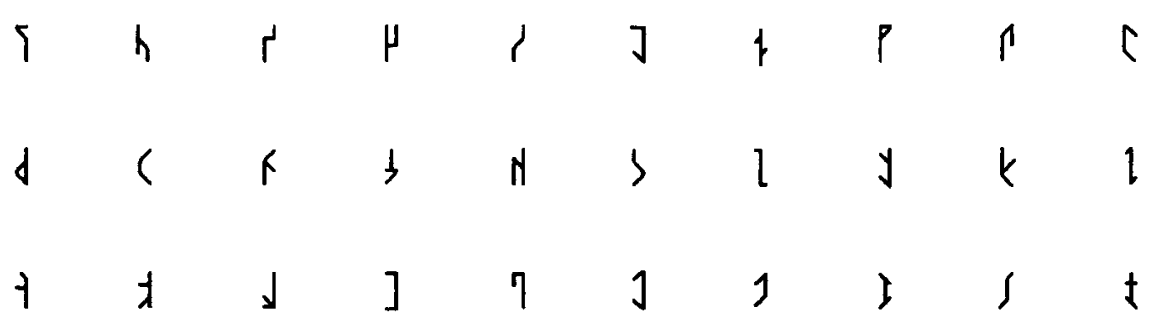

Figure 1. Examples of the novel shapes.

tions that they formed (end points, touching intersections, or one line touching between end points). In the instructions, participants were given examples of each junction and type of line.

For the visual identification test, participants were instructed that they would perform the same perception experiment as in the study phase, to compare the effects of practice on perception. They were presented with 30 shapes for $1 / 60 \mathrm{sec}$, with a backward mask of vertical, horizontal, and diagonal lines. The mask stayed on the screen for $2 \mathrm{sec}$ while the participants drew the shapes. Scoring of the drawings produced by the participants was conducted with the procedure used by Musen and Treisman (1990); each shape was scored correct if the lines were drawn to the correct orientation, and if the junctions were drawn correctly. For the visual episodic recognition test, the participants were presented with the 30 shapes for $3 \mathrm{sec}$ each and were asked to discriminate the studied shapes from the nonstudied shapes.

\section{Results}

As expected, visual identification of same-sized patterns was not different from identification of differentsized patterns $\left(F_{\mathrm{S}}<1\right.$; see Figure $\left.2 \mathrm{~A}\right)$. Reliable facilitation occurred for same-sized patterns, by subjects $\left[F_{1}(1,48)=\right.$ $\left.5.44, M S_{\mathrm{e}}=.02, p<.02\right]$, and by items $\left[F_{2}(1,48)=5.2\right.$, $\left.M S_{\mathrm{e}}=.02, p<.03\right] .{ }^{1}$ Reliable facilitation was also obtained for different-sized patterns $\left[F_{1}(1,48)=7.3, p<.01\right.$; $\left.F_{2}(1,48)=7.01, p<.01\right]$. However, visual identification of same-orientation patterns was reliably different from identification of different-orientation patterns $\left[F_{1}(1,48)=\right.$ $5.18, M S_{\mathrm{e}}=.02, p<.03 ; F_{2}(1,48)=4.00, M S_{\mathrm{e}}=.03, p<$ $.05]$. In fact, facilitation was obtained only for sameorientation patterns $\left[F_{1}(1,48)=11.02, p<.01 ; F_{2}(1,48)=\right.$ $6.85, p<.01]$, but not for different-orientation patterns $\left[F_{1}(1,48)=1.08 ; F_{2}<1\right]$, suggesting that visual identification of 2-D patterns is highly specific to orientation.

Consistent with prior findings, episodic recognition of visual patterns was reliably better for same-sized patterns than for different-sized patterns $\left[F_{1}(1,12)=5.81\right.$, $\left.M S_{\mathrm{e}}=.03, p<.03 ; F_{2}(1,24)=5.53, M S_{\mathrm{e}}=.05, p<.03\right]$. Similarly, recognition of same-orientation patterns was reliably better than that of different-orientation patterns $\left[F_{1}(1,12)=5.91, M S_{\mathrm{e}}=.02, p<.03 ; F_{2}(1,24)=6.12\right.$, $\left.M S_{\mathrm{e}}=.03, p<.02\right]$.

These findings are consistent with the transferappropriate processing view, and they suggest that the computational goals of identification can strongly influence the representation of perceptual information in memory. Contrary to prior reports of the lack of any adverse effect of size or orientation alterations on visual identification, the present experiment suggests an adverse ef- fect of a left-right orientation change, but not of a size change, on visual identification. Thus, it appears that left-right orientation information is included in the representations that mediate 2-D identification, because it is not an accidental property for identification of 2-D patterns (i.e., a change in orientation can cue a change in letter identity, as in $b$ vs. $d$ - see Roediger \& Srinivas, 1993; Srinivas, 1996b). In addition, episodic recognition of 2-D patterns was adversely affected by size and leftright orientation, supporting the view that episodic recognition depends on integrated representations of shape and perceptual information. In Experiment 2, we examined whether a similar pattern of results would be obtained for tactile identification and recognition.

\section{EXPERIMENT 2}

\section{Method}

Participants. One hundred twenty Boston College undergraduates participated in this experiment.

Materials and Design. The design was identical to that for Experiment 2, except that 30 participants each took part in tactile identification and recognition tests in the size and orientation change conditions. The materials were raised line drawings of the three-line patterns used in Experiment 1, constructed by embossing the shapes with a serrated wheel on plastic cards.

Procedure. At study, the participants tactually identified 20 shapes for $10 \mathrm{sec}$ each by providing a verbal description of the position, orientation, and intersections of the lines comprised by the shape. This was done to ensure that the participants would achieve at least $95 \%$ accuracy in tactile identification of the shapes. Each study session was preceded by practíce with two shapes. The items were not blocked by condition, and they were presented in a random order.

For the tactile identification test, the participants were presented with 30 shapes for $4 \mathrm{sec}$ each, with instructions similar to those in Experiment 1 . This time period was chosen on the basis of a pilot study with 30 participants that indicated a baserate performance on nonstudied items of about $30 \%$. Following presentation of the shapes for $4 \mathrm{sec}$, the participants were asked to draw the shape as accurately as possible, as in the visual identification test. For the episodic recognition test, the participants were presented the 30 shapes for $10 \mathrm{sec}$ each, and they were asked to discriminate the studied shapes from the nonstudied shapes.

\section{Results}

As is shown in Figure 2B, tactile identification appeared to depend on representations that included size and left-right orientation information. Identification of same-sized tactile patterns was reliably better than identification of different-sized patterns $\left[F_{1}(1,48)=3.77\right.$, 

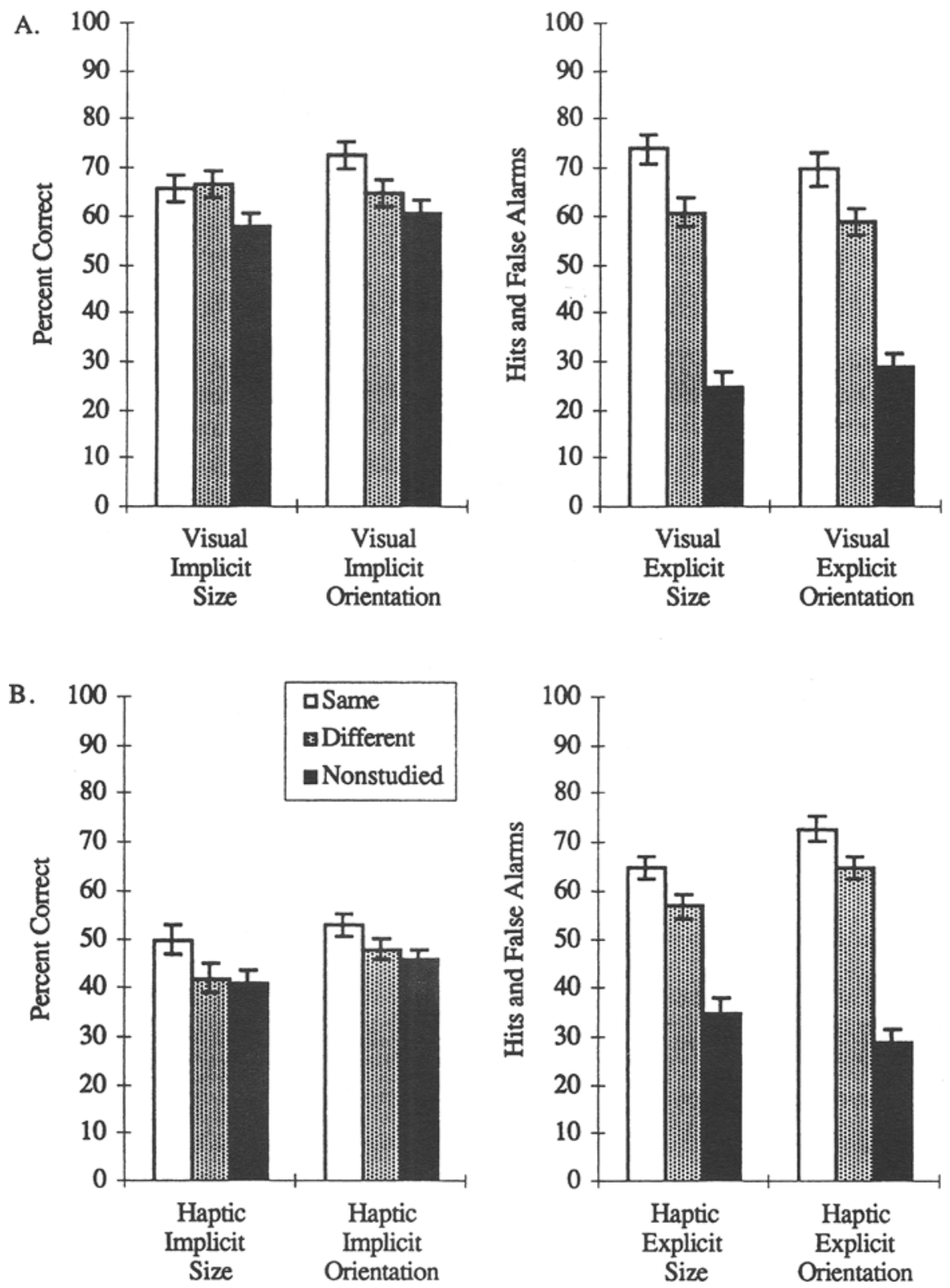

Figure 2. (A) Effects of size and orientation changes on visual identification and episodic recognition. (B) Effects of size and orientation changes on tactile identification and episodic recognition. $95 \%$ confidence intervals for the figures were based on the within-subjects $M S_{\mathrm{e}}$ recommended by Loftus and Masson (1994).

$\left.M S_{\mathrm{e}}=.02, p<.05 ; F_{2}(1,48)=4.64, M S_{\mathrm{e}}=.02, p<.03\right]$. Indeed, reliable facilitation was obtained for same-sized patterns $\left[F_{1}(1,48)=5.20, p<.02 ; F_{2}(1,48)=6.41, p<\right.$ $.01]$, but no facilitation occurred for different-sized patterns $(F \mathrm{~s}<1)$. A similar pattern emerged with orientation alterations. Identification of same-orientation patterns was reliably better than identification of differentorientation patterns $\left[F_{1}(1,72)=3.52, M S_{\mathrm{e}}=.02, p<.06\right.$; $\left.F_{2}(1,72)=3.67, M S_{\mathrm{e}}=.01, p<.06\right]$. Reliable facilitation relative to the nonstudied condition was obtained for sameorientation patterns $\left[F_{1}(1,72)=7.67, p<.01 ; F_{2}(1,72)=\right.$
$5.05, p<.03]$, but not for patterns changed in orientation $(F \mathrm{~s}<1)$.

As with visual recognition, episodic recognition of tactile patterns was mediated by representations that included size and left-right orientation information. Recognition of patterns in the same size was reliably better than recognition of patterns in a different size $\left[F_{1}(1,24)=\right.$ $4.05, M S_{\mathrm{e}}=.03, p<.05 ; F_{2}(1,24)=7.39, M S_{\mathrm{e}}=.01, p<$ $.01]$, as was recognition of patterns in the same orientation $\left[F_{1}(1,24)=6.13, M S_{\mathrm{e}}=.02, p<.02 ; F_{2}(1,24)=4.99\right.$, $\left.M S_{\mathrm{e}}=.02, p<.03\right]$. 
Once again, the tactile identification results were in support of the transfer-appropriate processing view, in that left-right orientation changes had an adverse effect on tactile identification. Size changes also had the same adverse effect on tactile identification, as was predicted by the transfer-appropriate processing approach. As discussed earlier, this result may simply reflect the fact that size changes are typically not related to distance changes in touch as they are in vision. ${ }^{2}$ Episodic recognition was dependent on representations that included size and orientation information, supporting the idea that recognition includes lower level perceptual information about objects that enables one to place them in a spatiotemporal context.

\section{DISCUSSION}

The results of this study suggest that the computational goals of the observer do in fact influence the representation of objects for identification. In Experiment 1, visual 2-D identification was unaffected by a size change as in prior research, but it was adversely affected by a change in left-right orientation. In Experiment 2, tactile 2-D identification was adversely affected by a change in size and by a change in left-right orientation. These findings are broadly consistent with the transfer-appropriate processing account of object representation (Roediger \& Srinivas, 1993; Srinivas, 1996b). According to transferappropriate processing, identification of 3-D objects is invariant across size and left-right orientation changes because these are accidental properties for 3-D object discrimination. Left-right orientation changes, however, are often nonaccidental properties for the discrimination of 2-D patterns, because they can cue a change in letter identity (a $q$ vs. a $p$ ). As a result, the transfer-appropriate processing approach predicts an effect of left-right orientation on visual and tactile 2-D identification, as was obtained in Experiments 1 and 2. Transfer-appropriate processing also predicts an adverse effect of size changes for tactile identification, because size change is not related to an arbitrary change in observer position in touch as it is in vision. Size changes are therefore not accidental changes for tactile identification in the same sense as they are for vision.

Interestingly, episodic recognition appears to be based on representations that include size and left-right orientation information, irrespective of the materials (2-D or 3-D) or modality through which an object is perceived (tactile or visual). These findings provide support for the view that episodic recognition is dependent on shape information along with lower-level perceptual information about the object.

One potentially troubling issue that these episodic recognition results raise is whether tactile identification as measured in these experiments was indeed tapping identification rather than episodic recognition. Because the results are similar for tactile identification and episodic recognition, this is a thorny problem. However, in an earlier study, we obtained a functional dissociation between tactile identification and episodic recognition with the exact same materials and procedures used in this study (Srinivas, Greene, \& Easton, 1997). Specifically, we found that tactile episodic recognition was enhanced by elaborative processing of the $2-D$ patterns at study, but that tactile identification was unaffected by elaboration. These results suggest that the similarities between tactile identification and episodic recognition reported here cannot easily be attributed to contamination by episodic memory.

Together, the findings on vision and touch suggest interesting similarities and differences between identification in the two modalities. Vision and touch appear to tap presemantic shape descriptions, because identification in both modalities is unaffected by elaborative processing at study (Hamman, 1996; Srinivas et al., 1997). Transfer across the two modalities is robust, implying that the two modalities share shape descriptions (Easton, Srinivas, \& Greene, 1997). The route to such shape description is somewhat different for the two modalities, however. For vision, size changes are discounted in the description of 2-D shapes, but for touch, size change does influence 2D shape descriptions.

\section{REFERENCES}

Biederman, I., \& Cooper, E. E. (1991). Evidence for complete translational and reflectional invariance in visual object priming. Perception, 20, 585-593.

BIEDERMAN, I., \& COOPER, E. E. (1992). Scale invariance in visual object priming. Journal of Experimental Psychology: Human Perception \& Performance, 18, 121-133.

Cave, C. B., Bost, P. R., \& CoBb, R. E. (1996). Effects of color and pattern on implicit and explicit picture memory. Journal of Experimental Psychology: Learning, Memory, \& Cognition, 22, 639-653.

CAVE, C. B., \& Squire, L. R. (1992). Intact and long-lasting repetition priming in amnesia. Journal of Experimental Psychology: Learning, Memory, \& Cognition, 18, 509-520.

Cooper, L. A., Schacter, D. L., Ballesteros, S., \& Moore, C. (1992). Priming and recognition of three-dimensional objects: Effects of size and reflection. Journal of Experimental Psychology: Leaining, Memory, \& Cognition, 18, 43-57.

EAston, R., SRINIVAS, K., \& GREenE, A. (1997). Do vision and haptics share common representations? Implicit and explicit memory within and between modalities. Journal of Experimental Psychology: Learning, Memory, \& Cognition, 23, 153-163.

Guarniero, G. (1974). Experience of tactile vision. Perception, 3, 101-104.

HamanN, S. B. (1996). Implicit memory in the tactile modality: Evidence from braille stem-completion in the blind. Psychological Science, 7, 284-288.

JOLICOEUR, P. (1987). A size-congruency effect in memory for visual shape. Memory \& Cognition, 15, 531-543.

Loftus, G. R., \& MASSON, M. E. J. (1994). Using confidence intervals in within-subject designs. Psychonomic Bulletin \& Review, 1, 476490.

Milliken, B., \& Jolicoeur, P. (1992). Size effects in visual recognition memory are determined by perceived size. Memory \& Cognition, 20, 83-95.

Musen, G., \& Treisman, A. (1990). Implicit and explicit memory for visual patterns. Journal of Experimental Psychology: Learning, Memory, \& Cognition, 16, 127-137. 
RoEdiger, H. L., III, \& SRINIVAS, K. (1993). Specificity of operations in perceptual priming. In P. Graf \& M. E. J. Masson (Eds.), Implicit memory: New directions in cognition, development and neuropsychology (pp. 17-48). Hillsdale, NJ: Erlbaum.

SCHaCter, D. L., CoOper, L. A., \& Delaney, S. M. (1990). Implicit memory for unfamiliar objects depends on access to structural descriptions. Journal of Experimental Psychology: General, 119, 5-24.

SRINIVAS, K. (1996a). Contrast and illumination effects on explicit and implicit measures of memory. Journal of Experimental Psychology: Learning, Memory, \& Cognition, 22, 1123-1135.

SRINIVAS, K. (1996b). Size and reflection effects in priming: A test of transfer-appropriate processing. Memory \& Cognition, 24, 441-452.

Srinivas, K., Breedin, S., Coslett, B. H., \& Saffran, E. (1997). Intact perceptual priming in a patient with bilateral damage to the inferior temporal lobes. Journal of Cognitive Neuroscience, 9, 490511.

Srinivas, K., Greene, A. J., \& Easton, R. D. (1997). Implicit and explicit memory for haptically experienced 2D patterns. Psychological Science, 8, 243-247.

White, B. W., Saunders, F. A., Scadden, L., Bach-Y-Rita, P., \&
Collins, C. C. (1970). Seeing with the skin. Perception \& Psychophysics, 7, 23-27.

\section{NOTES}

1. $M S_{\mathrm{e}} \mathrm{s}$ are based on an overall $F$ test with three levels of study condition as a within-subjects factor, and six levels of lists used for counterbalancing as a between-subjects factor.

2 . The finding that size changes adversely affect tactile identification is on its face inconsistent with prior reports of size constancy in tactile identification after limited practice (Guarniero, 1974; White, Saunders, Scadden, Bach-y-Rita, \& Collins, 1970). We note, however, that in these earlier experiments, participants demonstrated size constancy in tactile perception when they were computing the distance of objects. The issue addressed in the present experiment is somewhat different and is related to whether objects presented simply for tactile identification (as opposed to distance computation) are represented in a size-invariant manner in memory.

(Manuscript received October 18, 1996; revision accepted for publication May 22, 1997.) 